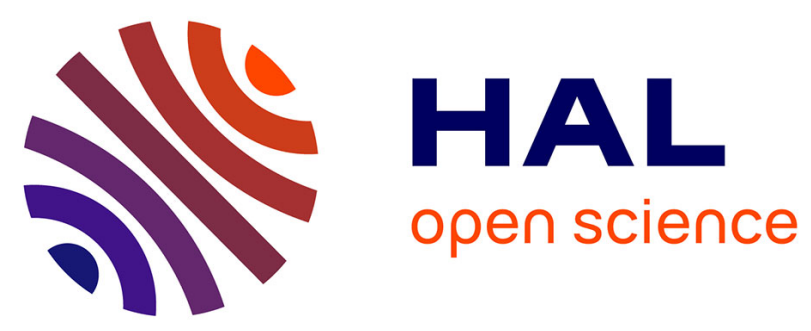

\title{
Interactive Manipulation Between a Human and a Humanoid: When Robots Control Human Arm Motion
}

Bruno Vilhena Adorno, Antonio Bo, Philippe Fraisse

\section{To cite this version:}

Bruno Vilhena Adorno, Antonio Bo, Philippe Fraisse. Interactive Manipulation Between a Human and a Humanoid: When Robots Control Human Arm Motion. IROS: Intelligent RObots and Systems, Sep 2011, San Francisco, United States. pp.4658-4663, 10.1109/IROS.2011.6048554 • lirmm-00641907

\section{HAL Id: lirmm-00641907 https://hal-lirmm.ccsd.cnrs.fr/lirmm-00641907}

Submitted on 16 Nov 2011

HAL is a multi-disciplinary open access archive for the deposit and dissemination of scientific research documents, whether they are published or not. The documents may come from teaching and research institutions in France or abroad, or from public or private research centers.
L'archive ouverte pluridisciplinaire HAL, est destinée au dépôt et à la diffusion de documents scientifiques de niveau recherche, publiés ou non, émanant des établissements d'enseignement et de recherche français ou étrangers, des laboratoires publics ou privés. 


\title{
Interactive Manipulation Between a Human and a Humanoid: When Robots Control Human Arm Motion
}

\author{
Bruno Vilhena Adorno, Antônio Padilha Lanari Bó, and Philippe Fraisse
}

\begin{abstract}
In this paper we present a novel approach in human/robot collaboration, where the robot controls not only its arm but also the human's by means of functional electrical stimulation (FES). The task is described by using the cooperative dual task-space approach, providing a considerable degree of invariance with respect to the morphology of the agents involved. Experimental results in a "ball in the hoop" task using healthy blindfolded subjects show the validity of the approach and encourages further experiments with impaired subjects, for instance hemiplegic or quadriplegic patients.
\end{abstract}

\section{INTRODUCTION}

The dream of having robots helping humans has a long history and is deeply inside the imagination of the modern society. Usually, in the imagined scenario robots have a huge capability in the interaction with humans.

For robots closely interacting with humans and/or in human environments, anthropomorphic structures always had an elevated status when compared to the nonanthropomorphic counterparts. One of the reasons is that "human tools match human dexterity" [1]. It means that humans design and build environments and tools suitable for human beings. Thus, one might expect that the more similar to a human the robot is, less modifications will be needed in the current environment and/or tools in order of using the robot effectively. Furthermore, humanoids and anthropomorphic designs can facilitate the human/robot interaction, because people are familiarized to work with other people [1].

In this paper, we are interested in physical human/robot collaboration, particularly those tasks where the robot assists explicitly the human, either an unimpaired person or a patient suffering from motor disabilities, such as movement disorders after stroke. In an important class of applications, the cooperation is defined such that the human holds complete control of the robot motion. Some examples in this class range from teleoperated robots to devices controlled by different forms of force control, such as [2]. On the other hand, in another less common class of applications the robot may directly affect human motion. For instance, in some applications shared control of the tasks exists, as often occurs in rehabilitation robotics [3]. The activation of human muscles in order to perform this shared control may also be conducted with the use of functional electrical stimulation (FES) [4]. Another possibility is the case where

This work is supported by Agence Nationale de la Recherche (ANR) under grant ANR 07-ROBO-011 in the context of the ASSIST project.

B. V. Adorno, A.P. L. Bó, P. Fraisse are with the University of Montpellier 2 and the (LIRMM), UMR 5506 - CC 477161 rue Ada, 34392 Montpellier Cedex 5 - France. E-mail: \{adorno, bo, fraisse\} alirmm.fr. the robot controls directly the human motion. This class of applications is intended to help patients suffering from severe motor disabilities, such as quadriplegic patients. In this work, we explore this new paradigm in human/robot collaboration, particularly using FES to induce human motion.

\section{A. Contributions and organization of the paper}

In this paper we extend our previous framework [5] to the case where the robot controls both its arm and the human's in order to maintain a coordination/collaboration. A "ball in the hoop" task is performed with five blindfolded healthy subjects in order to verify the two initial hypotheses: that it is possible to the robot to coordinate both human and robot arms; and that the collaboration will improve the task execution.

The paper is organized as follows: §II describes the cooperative task in terms of the cooperative dual taskspace framework. §III presents the control laws for the human arm in both joint and Cartesian spaces, and also the control laws for the robot arm and for the cooperative task. §IV describes the methodology used to perform the experiments. The experimental results, obtained by using five healthy blindfolded subjects, are presented in $\S \mathrm{V}$. Finally, $\S$ VI presents the conclusions of our study and the final remarks.

\section{DESCRIPTION OF THE COOPERATIVE TASK}

As shown in [5], the human/robot cooperative task can be effectively represented by the cooperative dual taskspace framework [6]. In this approach, rigid motions are represented by dual quaternions and the cooperative task can be represented by means of the relative configuration between the two hands. More specifically, the robot hand is represented by the dual quaternion $\underline{\mathbf{q}}_{R}$, the human hand is represented by $\underline{\mathbf{q}}_{H}$ and the task is given by

$$
\underline{\mathbf{q}}_{\mathrm{task}}=\underline{\mathbf{q}}_{R}^{*} \underline{\mathbf{q}}_{H},
$$

where $\mathbf{q}^{*}$ is the conjugate of the dual quaternion and both $\underline{\mathbf{q}}_{R}$ and $\underline{\mathbf{q}}_{H}$ are given with respect to a common coordinate system.

As we can see in (1), $\underline{\mathbf{q}}_{\mathrm{task}}$ represents the geometrical relation between the hands, and thus the goal is to control the system such that $\underline{\mathbf{q}}_{R_{m}}^{*} \underline{\mathbf{q}}_{H_{m}}$ (i.e., the relationship between the measured poses) converges to $\underline{\mathbf{q}}_{\mathrm{task}}$, meaning that the hands achieved the desired relative pose.

For the purpose of verifying the main hypothesis that the robot can control the human arm and achieve a collaboration, in this paper we defined a simple task where the robot holds a 


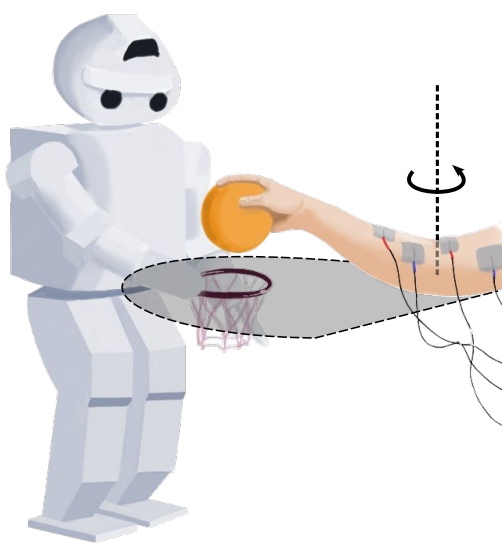

Fig. 1: Human/robot collaborative task: "ball in the hoop". The human arm is actuated by functional electrical stimulation and is constrained to move on a plane.

miniature basketball hoop and the person holds a ball. Then, the human arm - controlled by means of functional electrical stimulation - and the robot arm must be coordinated in order to drop the ball inside the hoop. To simplify the experimental setup and avoid the requirements of precise multi-joint FES control, the human arm is constrained to move only on a plane, as depicted in Fig. 1.

An interesting fact is that the addition of the human arm control does not change the definition of the task. In fact, whenever the task is represented by (1), there is an invariance with respect to which agents are controlled and even with respect to the controlled degrees of freedom (DOF).

This invariance can be useful when developing general assistant robots, because the task is defined only once and then the robot can interact with several other entities, regardless if these entities are other robots or people. In the case of healthy people, the robot would control only itself. In the case of people suffering from motor disabilities (e.g., quadriplegia), the robot could control the person's arm if otherwise it would be impossible to accomplish the taskfor example, when the robot cannot reach the person's hand without controlling the human $\operatorname{arm}^{1}$.

\section{CONTROL LAWS}

Once the task is defined in terms of $\underline{\mathbf{q}}_{\text {task }}$, the overall system is hierarchically controlled. First, there is the cooperation level responsible for making the measured task variablethat is, the measured relative configuration between the hands-converge to $\underline{\mathbf{q}}_{\text {task }}$. This level then calculates the reference signal in Cartesian space for both human's and robot's arms. Then, by means of a Jacobian based method, the references in the Cartesian space are transformed to control signals in joint space for both arms. In the following each controller is described in detail.

\footnotetext{
${ }^{1}$ Of course, it does not mean that the robot will control the subject's arm against his/her will. The robot performs only the low level control of the human arm whereas ultimately the person could somehow provide high-level commands (e.g., voice commands) to change or abort the task.
}

\section{A. Robot arm control}

Since our system is already represented in dual quaternion space, we can directly apply the following control law [7]:

$$
\dot{\vec{\theta}}_{R}=\mathbf{J}_{R}^{\dagger} \mathbf{K}\left({\underline{\mathbf{q}_{H_{m}}^{T}}}_{\underline{\mathbf{q}}_{\text {task }}^{*}-\underline{\mathbf{q}}_{R_{m}}^{T}}^{\longrightarrow}\right),
$$

where $\vec{\theta}_{R}$ is the vector containing the joint variables, $\mathbf{J}^{\dagger}=$ $\mathbf{J}^{T}\left(\mathbf{J J}^{T}+\lambda \mathbf{I}\right)^{-1}$ is the damped least-square inverse [8], $\mathbf{K}$ is a positive definite gain matrix and $\underline{\mathbf{q}}_{H_{m}}^{T}$ and $\underline{\mathbf{q}}_{R_{m}}^{T}$ are the measured poses of the human's and robot's hands (both with respect to the robot's torso), respectively, and $\mathbf{J}_{R}$ is the analytical Jacobian which satisfies $\underline{\overrightarrow{\mathbf{q}}}_{R}=\mathbf{J}_{R} \dot{\vec{\theta}}_{R}$ [7] and the upper arrow (e.g., $\overrightarrow{\mathbf{q}}$ ) represents the mapping of the dual quaternion $\mathbf{q}$ into $\mathbb{R}^{8}$. Then $\dot{\vec{\theta}}_{R}$ is passed directly to the robot built-in PID joint controllers.

\section{B. Human arm control using FES}

Functional electrical stimulation is the application of electrical pulses to neural pathways or directly to muscles, in order to provide functional benefit to the patient, typically by restoring lost or impaired neuromuscular functions. In order to meet this goal, FES systems attempt to mimic neural excitation, which is based on trains of action potentials. Hence, FES signals are based on trains of impulses, which are applied on the body by means of electrodes.

In spite of its great potential for different applications, the use of FES to satisfactorily control muscle action is still a major challenge [9], particularly when using superficial electrodes. Some of the difficulties involved are related to the diffusion of the stimulation to other muscles and the inherent complexity of muscle dynamics. For this reason, in the current experiment the FES-induced human arm motion is rather simple, limited to the control of the elbow angle and the opening of the hand. In order to control the elbow, only the Biceps Brachii (BB) is stimulated, which means that our FES system is not able to extend the elbow. To open the hand, mainly the Extensor Digitorum Communis (EDC) is activated. The placement of the electrodes is shown in Fig. 2.

For both stimulated muscles, an initialization procedure is required in order to set the appropriate FES parameters for a particular subject. In this procedure, the stimulation frequency, intensity and pulse width (PW) are chosen based on the obtained response and on the subject's subjective evaluation. The first two parameters are kept fixed during the experiment, while the stimulation PW is used to control the joint motion. In order to standardize the controller for different subjects, the control signal is first normalized based on the PW range defined by the subject.

Concerning the controller designed to control the elbow position, in this work we have used a simple PI controller with anti-windup. The latter feature is due to the actuator saturation with respect to physiological limits and subject comfort. The controller gains were set in order to minimize possible overshoots, since elbow extension is not actuated. A similar FES controller has already been applied for other applications, such as [10]. 


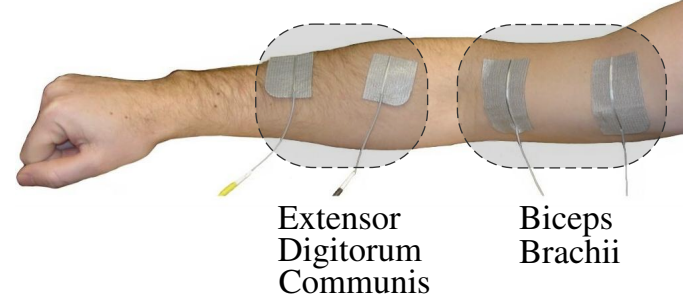

Fig. 2: Actuation of the human arm: positioning of the electrodes.

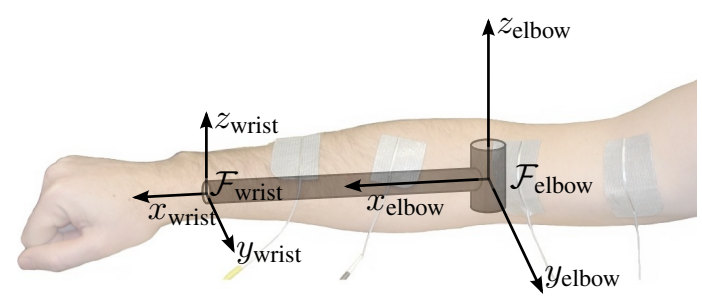

Fig. 3: The human arm modeled as a one-link serial robot.

\section{Human arm control in the Cartesian space}

In order to control the human arm in the Cartesian space, we consider the human arm as a serial manipulator such that

$$
\dot{\overrightarrow{\mathbf{q}}}_{H}=\mathbf{J}_{H} \dot{\vec{\theta}}_{H},
$$

where $\vec{\theta}_{H}$ is the vector containing the actuated joints of the human arm and $\underline{\mathbf{q}}_{H}$ is the rigid transformation of the hand with respect to a convenient coordinate system.

In this paper we consider only the actuation of the biceps and an on/off control of the wrist. In this manner, the human arm is considered as an one-DOF serial robot with the rotation axis located at the elbow, as illustrated in Fig. 3 and with Denavit-Hartenberg parameters shown in Table I. In this manner, the forward kinematic model-obtained directly in dual quaternion space [7] - relates the movement of the wrist with respect to the elbow. Hence (3) reduces to

$$
\dot{\overrightarrow{\mathrm{q}}}_{\text {wrist }}^{\text {elbow }}=\mathbf{J}_{\text {wrist }}^{\text {elbow }} \dot{\theta}_{H} \text {. }
$$

As we can see in (1), both human's and robot's hands are given with respect to a common frame. For convenience's sake, the reference frame for the cooperative task is the robot's torso. Furthermore, (4) provides the relation between the wrist and elbow, but there is a displacement between the wrist and the hand, given by $\underline{\mathbf{q}}_{H}^{\text {wrist. }}$. Thus, $\underline{\mathbf{q}}_{H}^{T}=$ $\underline{\mathbf{q}}_{\text {elbow }}^{T} \underline{\mathbf{q}}_{\text {wrist }}^{\text {elbow }} \underline{\mathbf{q}}_{H}^{\text {wrist }}$. In our experiment, we assume that $\underline{\mathbf{q}}_{\text {elbow }}^{T}$ and $\underline{\mathbf{q}}_{H}^{\text {wrist }}$ are constant, hence

$$
\begin{aligned}
& \underline{\dot{\mathbf{q}}}_{H}^{T}=\underline{\mathbf{q}}_{\text {elbow }}^{T} \underline{\dot{\mathbf{q}}}_{\text {wrist }}^{\text {elbow }} \underline{\mathbf{q}}_{H}^{\text {wrist }} \\
& \underline{\dot{\mathbf{q}}}_{H}^{T}=\stackrel{+}{\mathbf{H}}\left(\underline{\mathbf{q}}_{\text {elbow }}^{T}\right) \overline{\mathbf{H}}\left(\underline{\mathbf{q}}_{H}^{\text {wrist }}\right) \overrightarrow{\dot{\mathbf{q}}}_{\text {wrist }}^{\text {elbo }},
\end{aligned}
$$

where $\stackrel{+}{\mathbf{H}}(\cdot)$ and $\overline{\mathbf{H}}(\cdot)$ are the Hamilton operators [11] which
TABLE I: Denavit-Hartenberg parameters for the human arm.

\begin{tabular}{lllcc}
\hline link & $\theta$ & $d$ & $a$ & $\alpha$ \\
\hline 1 & 0 & 0 & 0.3 & 0 \\
\hline
\end{tabular}

satisfy $\stackrel{+}{\mathbf{H}}\left(\underline{\mathbf{q}}_{a}\right) \underline{\mathbf{q}}_{b}=\overline{\mathbf{H}}\left(\underline{\mathbf{q}}_{b}\right) \underline{\mathbf{q}}_{a}=\underline{\mathbf{q}}_{a} \underline{\mathbf{q}}_{b}$. Eq. (5) becomes

$$
\underline{\dot{\mathbf{q}}}_{H}^{T}=\underbrace{=\stackrel{+}{\mathbf{H}}\left(\underline{\mathbf{q}}_{\text {elbow }}^{T}\right) \overline{\mathbf{H}}\left(\underline{\mathbf{q}}_{H}^{\text {wrist }}\right) \mathbf{J}_{\text {wrist }}^{\text {elbow }}}_{\mathbf{J}_{H}^{T}} \dot{\theta}_{H} .
$$

The system represented in (6) is under-actuated, since the dual position control law requires six DOF, but only one DOF is actuated. In order to tackle this problem, only the translation part of $\underline{\mathbf{q}}_{H}^{T}$ is controlled. However, because the task is defined by the relative pose between the hands, the robot will automatically reconfigure its hand's pose to compensate the inability of controlling the human hand's rotation. The control law for the human arm is given by

$$
\dot{\theta}_{H}=\mathbf{J}_{\mathbf{t}_{H}^{T}}^{\dagger} \mathbf{K}\left(\overline{\mathbf{t}_{H_{d}}^{T}-\mathbf{t}_{H_{m}}^{T}}\right),
$$

where $\mathbf{t}_{H}^{T}$ is the pure quaternion (real part equals zero) representing the translation of the human hand with respect to the robot's torso, $\mathbf{K}$ is a positive definite gain matrix and $\mathbf{J}_{\mathbf{t}_{H}^{T}}$ is given by [6]:

$$
\mathbf{J}_{\mathbf{t}_{H}^{T}}=2 \overline{\mathbf{H}}\left(\mathbf{q}_{H}^{T *}\right) \mathbf{J}_{\mathbf{q}_{H}^{T^{\prime}}}+2 \stackrel{+}{\mathbf{H}}\left(\mathbf{q}_{H}^{T^{\prime}}\right) \mathbf{J}_{\mathbf{q}_{H}^{T}}^{*} .
$$

\section{Cooperative task control}

The ideal situation to perform the cooperative task control would be to have at least full actuation in both human and robot arms, that is, six-DOF in each arm. In this case, several strategies could be used in order to perform the cooperative task [6]. However, as we could see in §§III-C, currently we control only one joint of the human arm, which would provide one DOF. Furthermore, since only the biceps is actuated, the actuation occurs just in one direction. Thus we need to use a simpler cooperative-task control law than the ones proposed in [6].

The control objective, which follows from (1), is given by $\underline{\mathbf{q}}_{R_{m}}^{T *} \underline{\mathbf{q}}_{H_{m}}^{T} \rightarrow \underline{\mathbf{q}}_{\text {task }}$. We define the control law for the cooperative task as follows. First the robot passes to the human arm's Cartesian controller a reference $\mathbf{t}_{H_{d}}^{T}$ such that the human arm enters in the robot's workspace-typically in a predefined region known to be suitable to accomplish the cooperative task, e.g., the center of the robot's workspace. Then (7) provides $\dot{\theta}_{H_{d}}$ which is used as the reference for the low level PI which controls the human elbow joint. Once the human hand enters in a region defined by $\left\|\overrightarrow{\mathbf{t}_{H_{d}}^{T}}-\overrightarrow{\mathbf{t}_{H_{m}}^{T}}\right\|<$ $d_{\text {coll }}$, the robot starts to move its arm using (2). Typically, $d_{\text {coll }}$ is used to avoid the robot trying to cooperate when the human hand is far from the cooperation region, and it was determined empirically. The cooperative task control algorithm is summarized in Algorithm 1. Note that $\delta_{\text {stop }}$ is a convenient stop criterion for the cooperative task: once the 


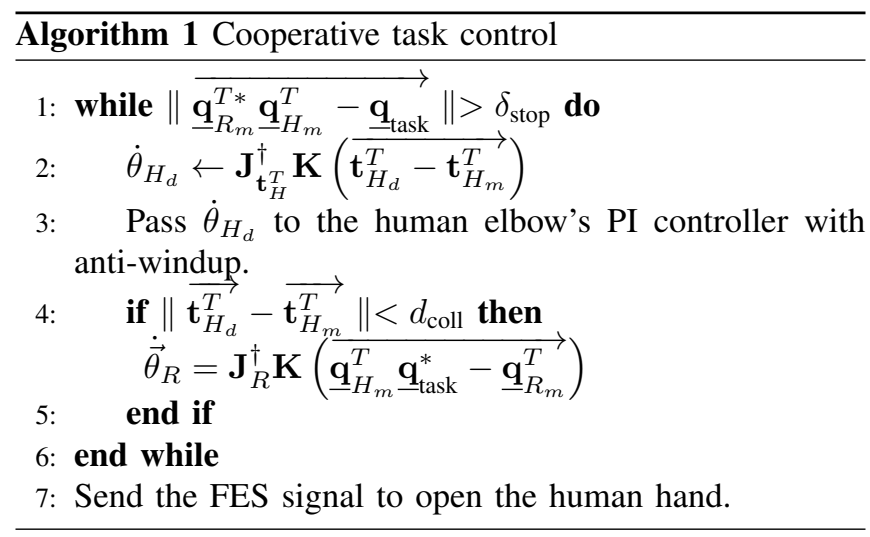

error in the task coordination is sufficiently small, the robot sends the FES signal to open the human hand.

\section{Methodology}

The task presented in §II may be divided in two subtasks. First, the reaching task must be performed, which is done by vertically aligning the arms, assuming that the human arm is higher than the robot's. Next, the human hand must be opened in order to drop the ball inside the hoop.

Because the reaching phase is characterized by the relative configuration between the basketball hoop and the ball, $\mathcal{F}_{H}$ and $\mathcal{F}_{R}$ represent the ball's and robot hand's coordinate systems, as illustrated in Fig. 4. The task, given by (1), can be assigned either geometrically or, as we will see in $\S \S I V-B$, it can be defined by means of a simple calibration process. In this calibration, we may also calculate and record some required information that is not available a priori.

\section{A. Experimental setup}

Five healthy volunteers participated in the experiment, three males and two females. They were positioned as shown in Fig. 6a, with the arms standing over a surface parallel to the robot's floor. Furthermore, the subjects were blindfolded in order to avoid the visual feedback, and thus to avoid any attempt to accomplish the task by natural actuation; that is, not by means of the functional electrical stimulation. In addition, they were asked to not move their bodies and to avoid the voluntary control of their arms - although they could not completely control their biceps, in principle they could disturb the task by controlling other muscles involved in the arm's movement. Another requirement imposed to the subjects was to maintain the same configuration between the wrist and the hand with the purpose of maintaining $\underline{\mathbf{q}}_{H}^{\text {wrist }}$ constant.

The hardware is composed by:

- a Fujitsu's HOAP-3 humanoid robot;

- a motion tracker - the Easytrack 500_-which consists of an active system based on linear cameras and an active marker which is typically mounted on the person's wrist or hand, or on the manipulated object;

- Prostim, a 8-channel stimulator designed jointly by the LIRMM and Neuromedics.
The only available measurements are $\underline{\mathbf{q}}_{M}^{E}$ and $\underline{\mathbf{q}}_{R}^{T}$. The former is the configuration of the active marker with respect to the Easytrack's coordinate system. The latter is obtained by using the robot's forward kinematic model in the dual quaternion space [7]. Since throughout the experiment (but not during the calibration process) the marker is fixed on the ball's surface, $\mathcal{F}_{H} \leftarrow \mathcal{F}_{M}$ and $\underline{\mathbf{q}}_{H}^{E}=\underline{\mathbf{q}}_{M}^{E}$.

\section{B. Calibration}

In order to simplify the experiment, we assume that the robot is fixed with respect to the motion tracker and that the subjects' elbows are located roughly at the same place and with the same configuration, as shown in Fig. 6a. Because $\underline{\mathbf{q}}_{H}^{E}$ and $\underline{\mathbf{q}}_{R}^{T}$ are given with respect to the motion tracker and the robot's torso, respectively, a calibration procedure is executed in order to find the relation between $\mathcal{F}_{E}$ and $\mathcal{F}_{T}$; that is, $\underline{\mathbf{q}}_{T}^{E}$. We proceed as follows.

First the marker is put on the robot hand such that their coordinate systems coincide, i.e. $\mathcal{F}_{R} \leftarrow \mathcal{F}_{M}$, and the measure $\underline{\mathbf{q}}_{R_{0}}^{E}=\underline{\mathbf{q}}_{M}^{E}$ is stored. Then the marker is put on the object and the subject places his/her arm in the initial configuration while holding the object. In this case, $\mathcal{F}_{H} \leftarrow \mathcal{F}_{M}$, and the measure $\underline{\mathbf{q}}_{H_{0}}^{E}$ is stored.

The relation between $\mathcal{F}_{T}$ and $\mathcal{F}_{E}$ is then given by

$$
\underline{\mathbf{q}}_{T}^{E}=\underline{\mathbf{q}}_{R_{0}}^{E}\left(\underline{\mathbf{q}}_{R_{0}}^{T}\right)^{*} .
$$

The procedure is repeated in the same manner with the subject's elbow and wrist to obtain $\underline{\mathbf{q}}_{\text {elbow }}^{E}$ and $\underline{\mathbf{q}}_{\text {wrist }}^{E}$, respectively. The relation between the grasped object and the wrist is also considered constant and given by

$$
\underline{\mathbf{q}}_{\mathrm{wrist}}^{H}=\left(\underline{\mathbf{q}}_{H_{0}}^{E}\right)^{*} \underline{\mathbf{q}}_{\mathrm{wrist}}^{E} .
$$

Finally, in order to define the task, the subject's hand is positioned with respect to the robot hand and the measure $\underline{\mathbf{q}}_{H_{\text {task }}}^{E}$ is stored. Because the desired task is also constant and defined in terms of relative dual position between the robot hand and the grasped object, then

$$
\underline{\mathbf{q}}_{\mathrm{task}}=\left(\underline{\mathbf{q}}_{R_{0}}^{E}\right)^{*} \underline{\mathbf{q}}_{H_{\mathrm{task}}}^{E} .
$$

The calibration procedure, summarized in Algorithm 2, is performed just once and with just one subject.

\section{EXPERIMENTAL RESULTS}

The focus of this experimental evaluation is on the performance of the human/robot collaboration, not on the performance of the robot controller or the performance of FES control. We do not seek precision in the experiment, neither in robot control nor in human arm control, but we are interested in effective cooperation. In this manner, we adopted the following metrics to evaluate the cooperative task. A task was considered successfully accomplished whenever the ball hit the border or entered in the hoop, meaning that a good coordination was achieved. The robot's performance was considered "good" if the robot tried to reach the human hand in a pose suitable for the success of the task, otherwise its performance was considered "bad". 

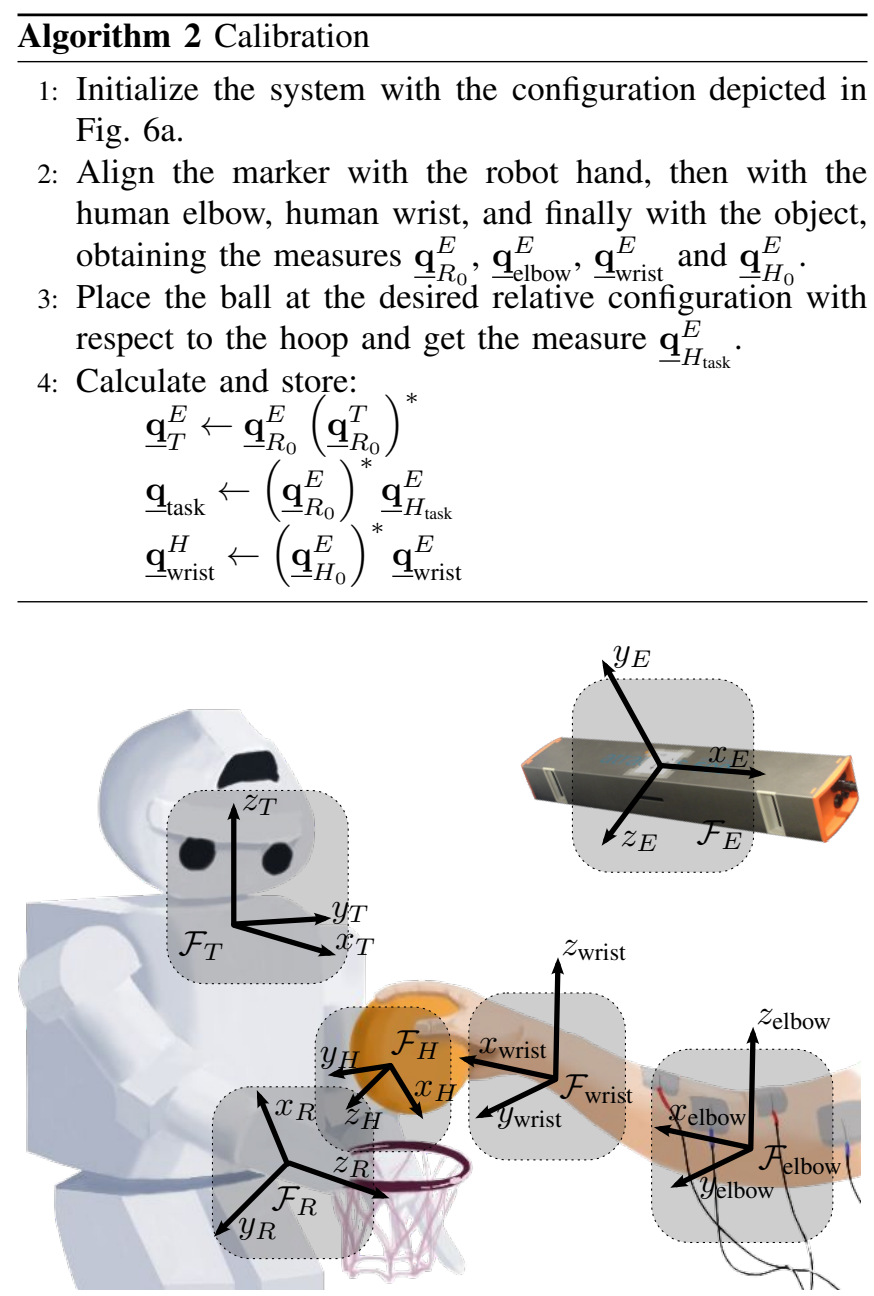

Fig. 4: Important coordinate systems defined in the experiment.

The human's performance-i.e., the result of the human arm control-was considered "good" whenever the human arm entered and stayed in the cooperation zone, that is, in the robot's workspace. When both agents-human and robot-had a good performance, we considered the task as an effective cooperation. In the following we present a detailed analysis of the experiments performed with five healthy blindfolded subjects.

Fig. 6 shows a successful cooperation sequence. The human arm enters in the cooperation zone in about one second, but the task cannot be accomplished because the robot is adjusting its arm's pose. Once their relative configuration is achieved, the FES signal is sent to the human forearm in order to open the hand. Because the agents cooperated, they could obtain a complete success. Fig. 5 shows the correspondent time responses for the human elbow joint and the control signal from the PI-with-anti-windup controller. For this particular subject, the cooperation workspace (the workspace where the cooperation is feasible) was given roughly when the human arm joint was between $30-50^{\circ}$. Because in order to obtain the human arm joint angle the robot uses the information of the marker's position, after the

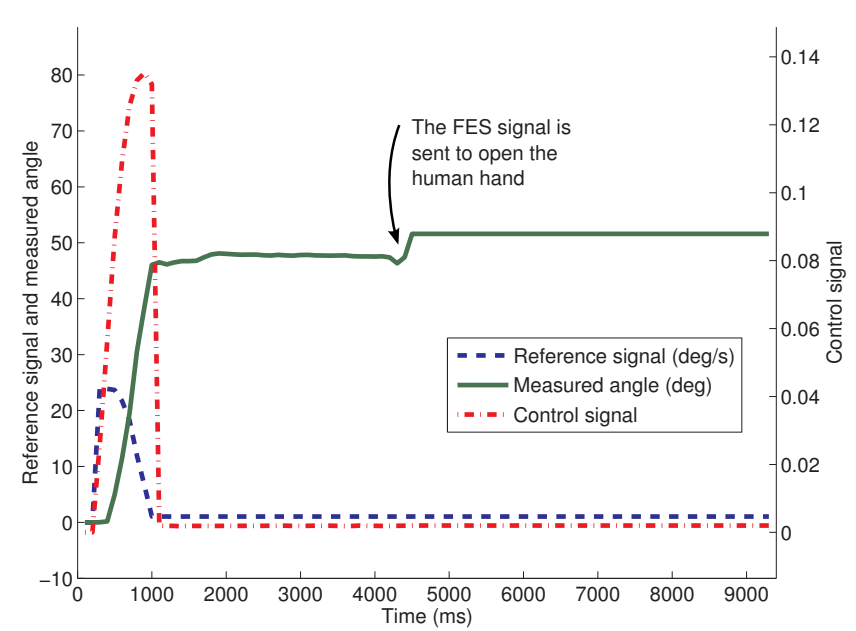

Fig. 5: Time response for the successful trial shown in Fig. 6.

ball falls the system stops measuring the angle of the human arm.

Fig. 7a shows the individual performance in the cooperative task. With the exception of Subject 2, in the total the robot had better performance, in terms of collaboration, than the FES controlled human arm. This was already expected since the human arm was actuated only in one direction and due to limitations on joint control using FES. Therefore, whenever the human arm left the cooperation zone, it was impossible to return. On the other hand, the robot's performance could be better if the ball's orientationi.e., the orientation of the marker attached to the ball-had not been considered in the task, or if the robot had more degrees of freedom.

Fig. 7b shows the impact of the effective cooperation in the success of the task. Whenever there is an effective cooperation, the number of successes tends to be close to the number of effective co-operations. This was also expected, since when both human and robot achieve good coordination, the error in the task execution tends to be smaller.

Finally, Fig. 7c shows the success rate when the effective collaboration is considered-that is, for each subject the number of successes is divided by the number of effective co-operations - or when the performance of the best agent is considered, i.e., for each subject the number of successes is divided by the best case shown in Fig. 7a. The success rate is higher when there is an effective collaboration than when one agent performs very good, but not necessarily the other one. It suggests that, instead of having one agent to be extremely good in order to compensate the other's error, the success rate is higher when both agents are "good enough".

\section{CONClusions}

In this paper we have presented a novel approach in human/robot collaboration where the robot controls both its arm and the human's. The latter is controlled by means of functional electrical stimulation. The task is defined in terms of the relative configuration between the human and robot 


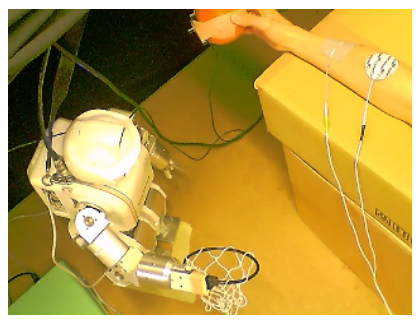

(a) $0 \mathrm{~s}$

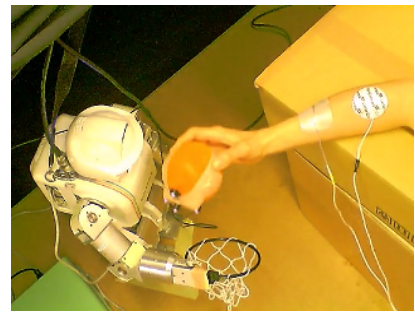

(b) $2 \mathrm{~s}$

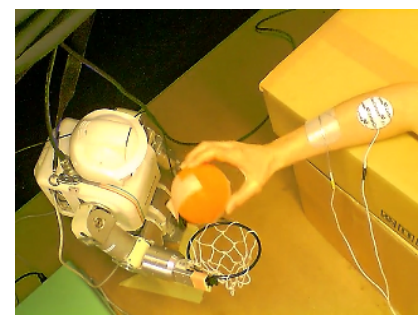

(c) $5 \mathrm{~s}$

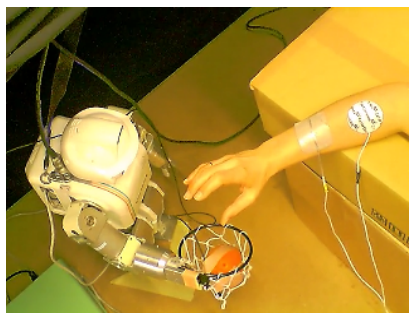

(d) $7 \mathrm{~s}$

Fig. 6: One cooperative task sequence for Subject 3.

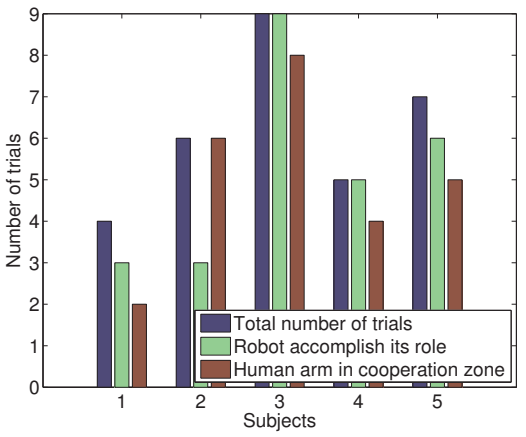

(a)

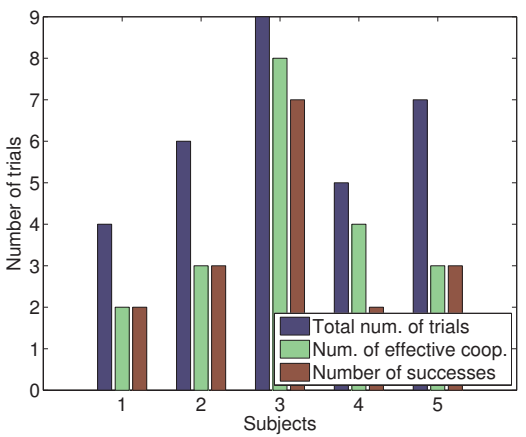

(b)

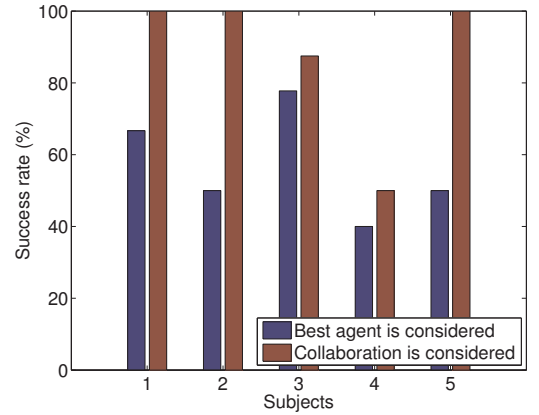

(c)

Fig. 7: Experimental results: (a) Number of trials versus individual performance; (b) Impact of the cooperation in the success; (c) Comparison of the success rate.

hands, hence providing a high degree of invariance with respect to the morphology of the agents involved. Two hypotheses were postulated and confirmed by an experimental evaluation using five healthy blindfolded subjects in a "ball in the hoop" task: first, the robot can control the human arm in order to achieve a desired coordination; second, a good coordination implies in a higher success rate when compared with just the best-collaborator's performance. In other words, two collaborators performing just "good enough" leads to higher success rate when compared to a very good cooperator interacting with a bad one. This has an important implication in assistive robotics, mainly when the robot must assist impaired people who cannot control their upper limbs. The FES control of the human arm need not to be precise, just good enough to reach the robot's workspace. Our next steps will be to control both biceps and triceps, leading to full elbow joint control. Furthermore, we are going to use a KUKA LWR robot, which has 7-DOF, to avoid the mechanical limitations of the robot used in this work. Also, we want to investigate suitable metrics to quantitatively analyze more general cooperative tasks. Encouraged by the experimental results, we also plan to perform human/robot collaboration experiments with hemiplegic or quadriplegic patients.

\section{ACKNOWLEDGMENT}

Thanks for Christine Azevedo, Mariana Bernardes and Pedro Moreira for being volunteers in the experiments.

\section{REFERENCES}

[1] C. C. Kemp, P. Fitzpatrick, H. Hirukawa, K. Yokoi, K. Harada, and Y. Matsumoto, Handbook of Robotics. Springer, 2008, ch. 56 Humano, pp. 1307-1333.

[2] P. Evrard, N. Mansard, O. Stasse, A. Kheddar, T. Schauss, C. Weber, A. Peer, and M. Buss, "Intercontinental, multimodal, wide-range telecooperation using a humanoid robot," in 2009 IEEE/RSJ Int. Conf. on Intelligent Robots and Systems, 2009, pp. 5635-5640.

[3] H. I. Krebs, N. Hogan, M. L. Aisen, and B. T. Volpe, "Robot-aided neurorehabilitation." IEEE Transactions on Rehabilitation Engineering, vol. 6, no. 1, pp. 75-87, Mar. 1998.

[4] C. Freeman, A. Hughes, J. Burridge, P. Chappell, P. Lewin, and E. Rogers, "Iterative learning control of FES applied to the upper extremity for rehabilitation," Control Engineering Practice, vol. 17, no. 3, pp. 368-381, Mar. 2009.

[5] B. V. Adorno, A. P. L. Bó, P. Fraisse, and P. Poignet, "Towards a Cooperative Framework for Interactive Manipulation Involving a Human and a Humanoid," in IEEE Int. Conf. on Robotics and Automation, 2011.

[6] B. V. Adorno, P. Fraisse, and S. Druon, "Dual position control strategies using the cooperative dual task-space framework," in IEEE/RSJ Int. Conf. on Intelligent Robots and Systems, 2010, pp. 3955-3960.

[7] H.-L. Pham, V. Perdereau, B. V. Adorno, and P. Fraisse, "Position and orientation control of robot manipulators using dual quaternion feedback," in 2010 IEEE/RSJ International Conference on Intelligent Robots and Systems. Taipei: IEEE, Oct. 2010, pp. 658-663.

[8] S. Chiaverini, "Singularity-robust task-priority redundancy resolution for real-time kinematic control of robot manipulators," IEEE Trans. on Robot. and Autom., vol. 13, no. 3, pp. 398-410, Jun. 1997.

[9] C. Lynch and M. Popovic, "Functional Electrical Stimulation," IEEE Control Systems Magazine, vol. 28, no. 2, pp. 40-50, Apr. 2008.

[10] A. Bó and P. Poignet, "Tremor attenuation using FES-based joint stiffness control," in 2010 IEEE International Conference on Robotics and Automation (ICRA). IEEE, 2010, pp. 2928-2933.

[11] B. Akyar, "Dual quaternions in spatial kinematics in an algebraic sense.” Turk. J. Math., vol. 32, no. 4, pp. 373-391, 2008. 